\title{
THE JOURNAL OF NeUrology AND Psychiatry
}

FOUNDED BY S. A. K. WILSON

\section{CEREBRAL VASCULAR LESIONS AND THE TENTORIAL PRESSURE CONE}

\author{
BY \\ R. S. ALLISON AND J. E. MORISON \\ From the Royal Victoria Hospital and the Queen's University Institute of Pathology, Belfast
}

(ReCEIVED 29TH December, 1939)

HeRniation through the incisura tentorii as the result of increased intracranial pressure has been emphasized recently by several observers, notably Geoffrey Jefferson (1938) and Moore and Stern (1938), as the anatomical basis for some organic nervous symptoms. This lesion is generally regarded as being especially prone to occur with abscesses involving the temporal lobe, but it has been noted in association with tumours of this region and of other parts of the brain. The present case is an example of such a herniation in which the structural changes have been especially studied, and in particular the altered relations of the posterior cerebral artery to the edge of the tentorium cerebelli.

\section{Clinical History}

\section{Case Report}

The patient, Kathleen B., was a child of ten years. The previous history contained nothing of note. Early in the month of September, 1938, the parents noticed she was becoming dull and listless. She was taken to a Welfare Centre but no definite conclusions were reached as to the cause of this change. On September 27th acute symptoms presented themselves in the form of intense headache and repeated vomiting. On the third day the parents took the child to the Belfast Hospital for Sick Children. On examination it was found that there was marked head retraction, a positive Kernig's sign and other evidence of meningeal irritation. Lumbar puncture was performed and the fluid was found to be turbid and under pressure. Protein was slightly increased ( 0.06 per cent.) and there were 500 cells (lymphocytes) per $\mathrm{cm}$. The sugar and chloride content was normal. Culture was sterile and the Wassermann reaction negative. Admission to hospital was urged but the parents refused.

An interval of 18 days elapsed. The child apparently improved to some extent, 
but on the 18th November the parents brought her back to hospital and she was admitted under the care of Dr. F. M. B. Allen. The clinical condition was then similar to that found at the previous examination with the addition, however, that the pupils were now unequal, the left being larger than the right. The cerebro-spinal fluid was still under pressure but contained only 40 lymphocytes per $\mathrm{cm}$. Congestion and swelling of the optic discs were observed, especially on the left side. The child exhibited noisy behaviour and great motor restlessness so that accurate observation was difficult. The following day she had a seizure, consciousness being lost and clonic convulsive movements occurring in the right arm and leg. After the fit the patient remained in a comatose state for twenty-four hours. When consciousness was regained she complained of pain in the left ear. The temperature, which had been normal, rose to $102^{\circ} \mathrm{F}$. Aural examination showed nothing definite. There was neither tenderness nor swelling over the mastoid process, but after consultation Mr. D. H. Craig exposed the middle ear and mastoid. The bony cortex was sclerotic, but beneath it, pus was found under pressure and there was a cholesteatomatous mass which was eroding the tegmen. The middle and posterior fossæ were exposed but the dura was found to be healthy. The general condition of the patient was so poor as to make further steps hazardous and the operation was concluded. The pus removed was cultured and gave a profuse growth of the staphylococcus aureus.

Following operation, the child remained semicomatose, but by the 26th November she had sufficiently improved to co-operate on examination. It was then apparent that she was blind, neither hand movements nor light being appreciated and the pupils remaining dilated and fixed. At this time the left plantar reflex was found to be extensor, but subsequently and until the time of her death both plantars were flexor. One of us (R. S. A.) was invited to see her on the 30th November. The abnormal signs observed then were as follows :

Psychic functions: The patient lay quietly on her side repeating over and over again: "Will you tell my daddy, please, my daddy please," she was able to understand what was said to her and reply correctly and could carry out simple requests. When given objects such as a hair brush, or when a pair of scissors was placed in her hand, she showed by gesture that she understood what they were. But when asked to name them she replied, "I know . . . a . . . a . . .". Mentioning the name of the object immediately secured her recognition.

Cranial nerves: Papillœdema was present, most marked on the left side. The presence of some bilateral secondary optic atrophy suggested that the condition had been present for some time. The pupils were dilated, immobile and unequal, and the left greater than the right. Upward movements of the eye were deficient on the left side but otherwise the ocular movements were full and there was no nystagmus. The right naso-labial fold was indistinct and weakness of the lower part of the right face was evident on voluntary and involuntary movements.

Reflexes: The abdominal reflexes were absent on the right side. The plantar reflexes were flexor in type. The knee and ankle jerks could not be obtained.

There was no obvious loss of power except in the face. Co-ordination was good and no sensory changes were observed. The signs of meningeal irritation formerly observed had disappeared. A diagnosis of temporal lobe abscess was made and craniotomy advised, but the patient became steadily worse, and coma and death supervened some days later.

\section{Post-Mortem Findings}

A post mortem examination was conducted within two hours of death. Permission was granted only to examine the cranial contents. The operation wound behind the left ear was discharging a little pus. On removal of the 
calvarium the dura was found taut and adherent to the underlying brain over the left temporal region. There were no organized thrombi in the cortical veins nor in the great venous sinuses. No abscess was found between the dura and the roof of the left middle ear. The left middle ear and the mastoid air cells contained only a small quantity of pus. The other air sinuses and the pituitary fossa were normal. No exudate was found over the base of the brain and no appreciable thickening of the membranes.

Over the left mid-temporal gyrus a pale yellowish grey area was seen which felt soft and fluctuant. On the medial aspect of the occipital lobe the cortex

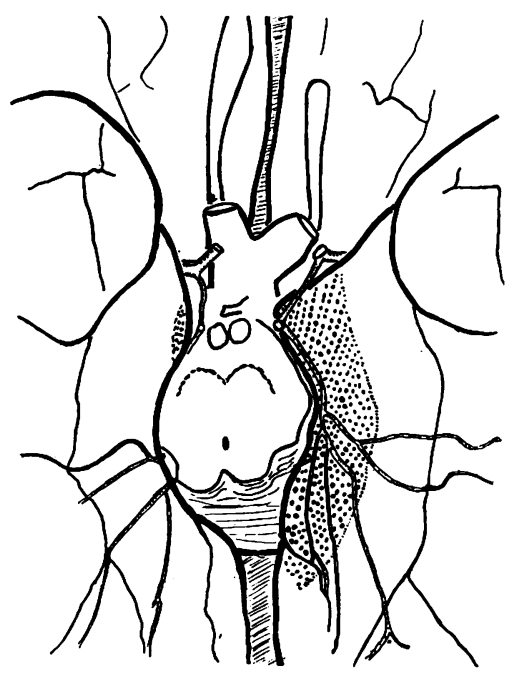

Fig. 1.-A line drawing traced over a photograph of the inferior surface of the brain. The left posterior cerebral artery is displaced on to the inferior aspect of the brain. Its branches may be pressed upon by the edge of the tentorium cerebelli or the vessel may be stretched or its branches kinked. The herniated area is dotted. Note the increase of the inferior surface medial to the collateral fissure.

above the calcarine fissure was shrunken and slightly yellow in colour. Below the fissure it was congested, slightly swollen and showed multiple petechial hæmorrhages. These changes did not extend to the tip of the occipital pole nor to the lateral aspect. The right occipital lobe was normal. The mid-line structures were noticeably displaced towards the right. The left uncus was clearly seen, the left hippocampal gyrus being grooved by the tentorial edge (Fig. 1). The posterior cerebral artery was stretched by the prolapsed gyri and lay in the hippocampal sulcus on its medial margin. The line of the tentorial edge crossed the course of the artery only in the extreme posterior part of its course and there was no evidence of thrombosis of the vessel or of its branches. On the right side the tentorium cerebelli had produced a tiny notch on the medial margin of the hippocampal gyrus where it turned round into the uncus. Owing to displacement of the brain to the right, the mid-brain at the level of the third cranial nerve had been deeply grooved on its lateral aspect by the right free margin of the tentorium cerebelli (Fig. 2). At this level the fourth cranial nerve swept round to the ventral aspect of the brain. There 
was very slight moulding of the medulla and of the cerebellar tonsils by the foramen magnum.

On section of the brain after fixation a large irregular shaped abscess was found occupying the greater part of the left temporal lobe (Fig. 3). This had extended forwards to within one inch of the anterior tip of the temporal lobe. More medially, another prolongation reached the posterior part of the lentiform nucleus and the œdematous wall came into relation with the more posterolateral and inferior part of the internal capsule. The medial wall of the abscess cavity was formed by the ependyma of the occluded inferior horn of the lateral ventricle. It was thus closely applied to the hippocampus and to the thrombosed

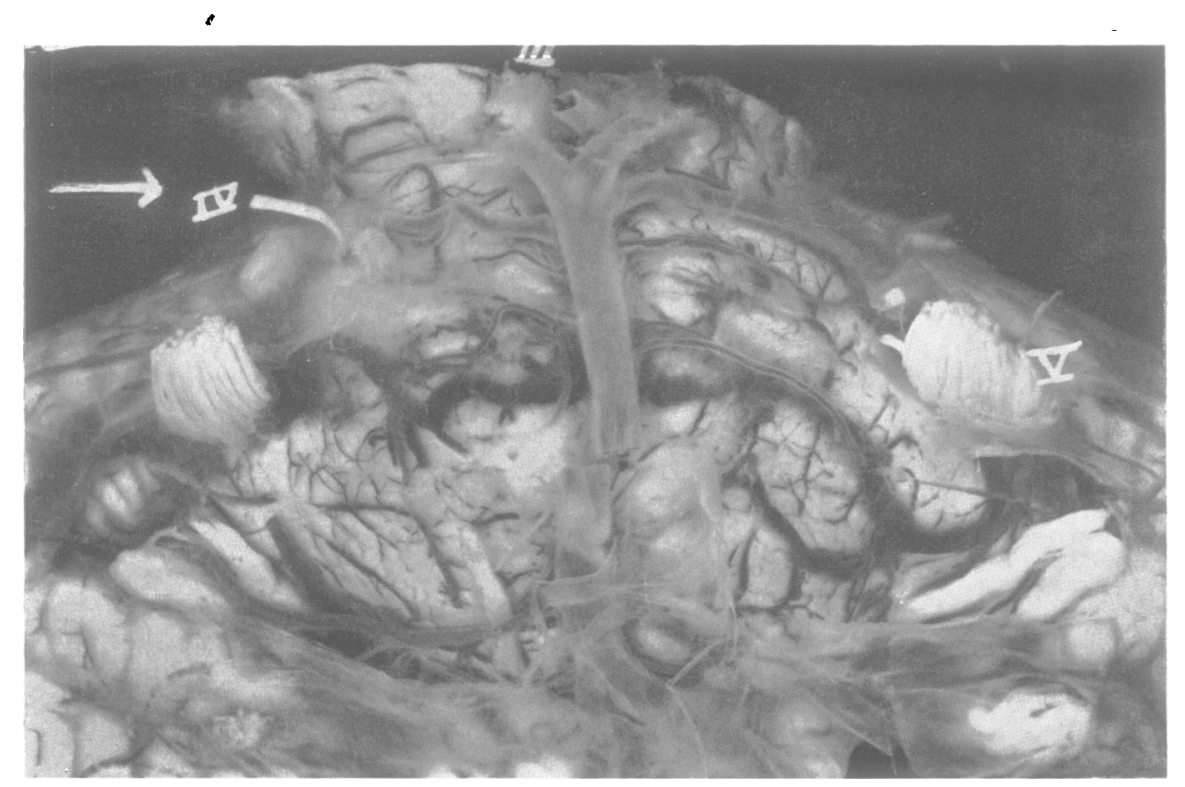

Fig. 2.-This shows the grooving of the right (contralateral) peduncle by the edge of the tentorium cerebelli. The cranial nerves are numbered.

choroid plexus of the inferior horn. A part of the uncus and of the hippocampal gyrus had been driven downwards through the incisura tentorii (Fig. 4). Posteriorly, about the level of the splenium, this abscess communicated with a thicker walled abscess which extended almost to the surface of the mid-temporal lobe and as far back as the upper limb of the mid-temporal sulcus. In addition to the two infarcts of different age in the occipital cortex there was a recent area of softening with areas of hæmorrhage in the intero-lateral part of the thalamus. In the prolapsed uncus of the left side there was a well marked band of hæmorrhage beneath the grey matter. There were a few small areas of softening and hamorrhage in the mid-brain on the right side.

A staphylococcus aureus was isolated from the left ear, the temporal lobe abscess and from a post-mortem specimen of the cerebro-spinal fluid. 


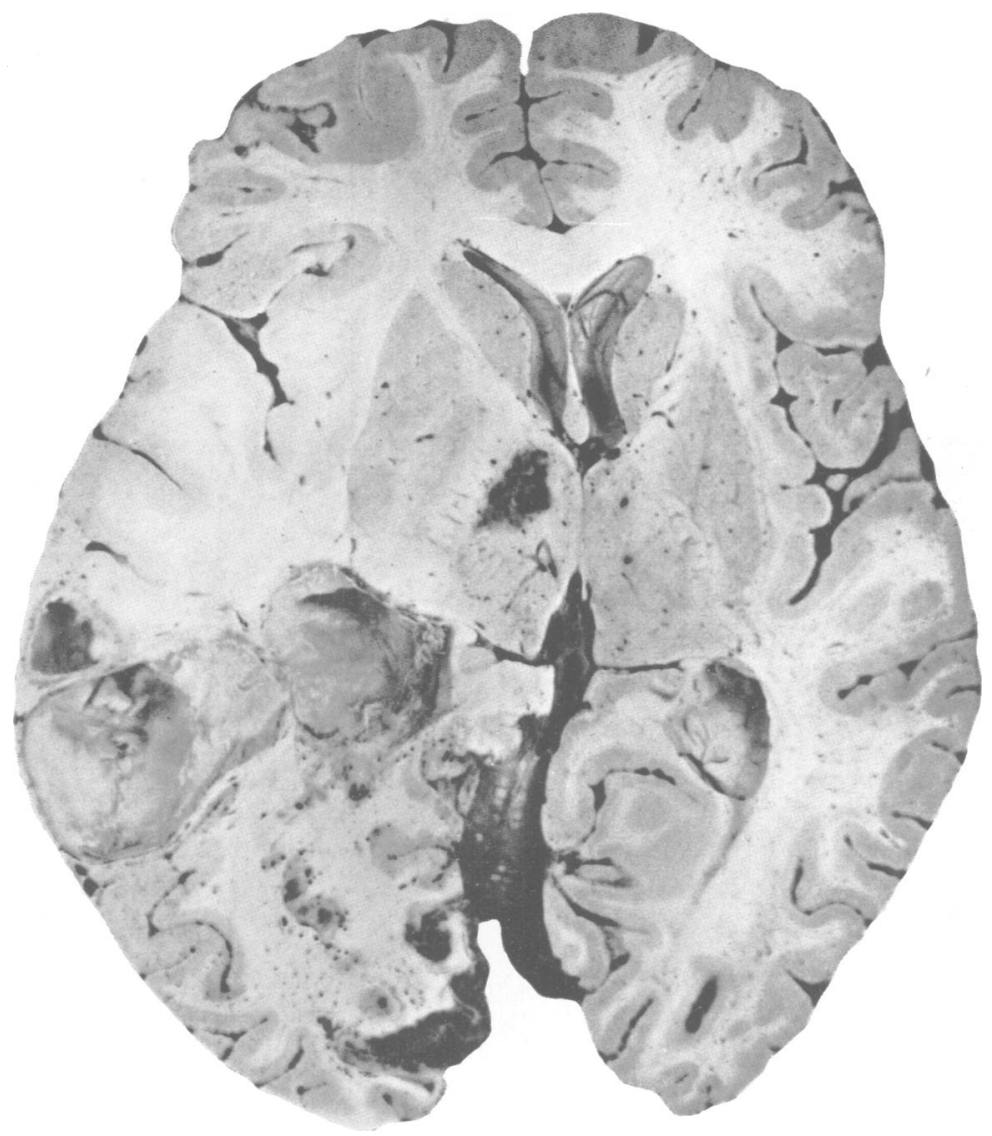

Fig. 3. - Section of the brain showing part of the abscess, the occipital infarct below the calcarine fissure and the thalamic infarct.

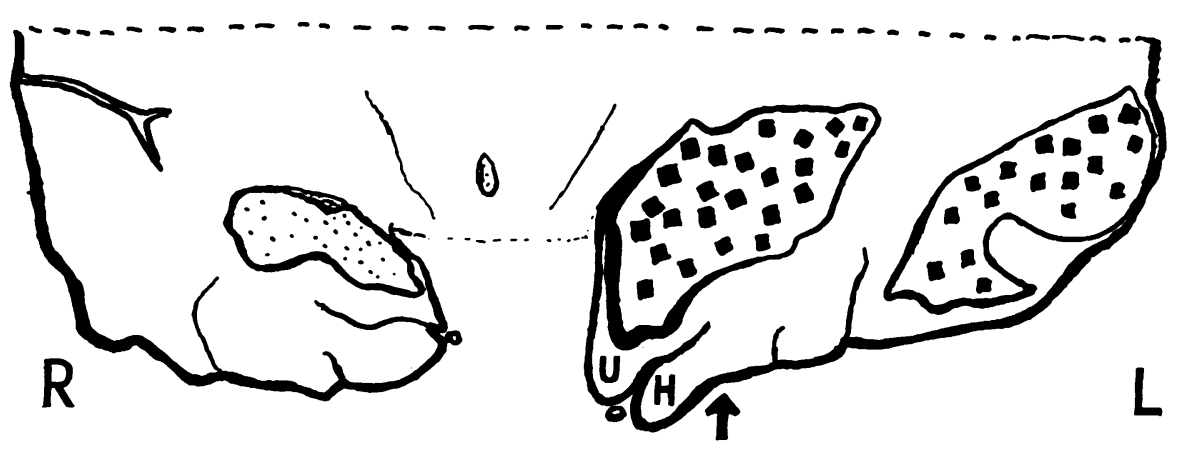

Tentorial

Groove

Fig. 4.-A line drawing of sections of brain showing tentorial groove and part of the uncas $(U)$ and of the hippocampal gyrus $(\mathrm{H})$ pressed downwards. 


\section{Microscopical Examination}

Sections of the temporal bone showed considerable fibroblastic proliferation and the inflammatory lesion was regressive. The pituitary was normal. The walls of the abscess were formed by granulation tissue infiltrated by plasma cells and lymphocytes. The capillary endothelial cells were swollen. Outside this there was evidence of considerable glial proliferation. Mononuclear cells, chiefly lymphocytes were present in some excess in the perivascular spaces and in the overlying subarachnoid space. Sections from the left occipital lobe in the area above the calcarine fissure showed the cortical grey matter, more

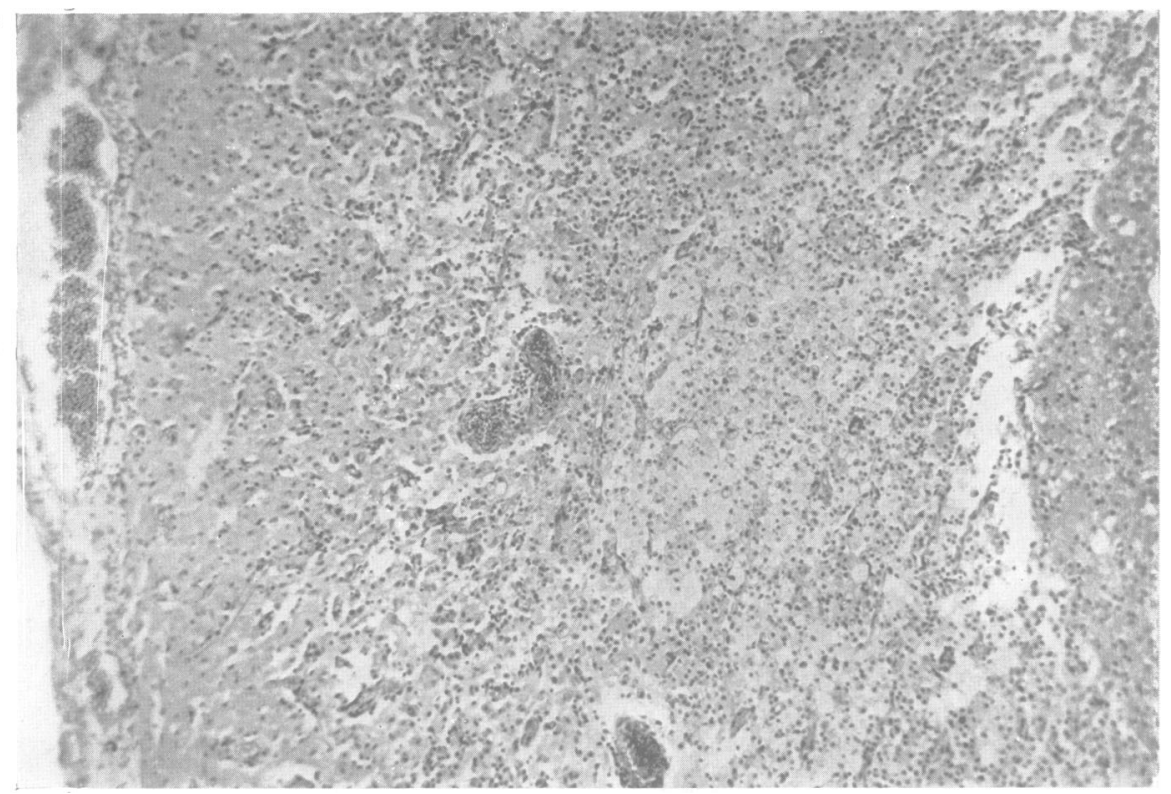

Fig. 5.-The cortex above the calcarine fissure. Fat engorged compound granular corpuscles, proliferation of microglia, and swelling of the capillary endothelial cells are most marked in the deeper layers.

especially in the deeper laminæ, in the process of removal by compound granuial corpuscles. These were gorged with fat. Astrocytes were proliferating. The blood-vessels were very prominent and the capillary endothelial cells greatly swollen (Fig. 5). In the lingual gyrus the changes were much more recent. The capillary endothelium was swollen and from many capillaries red blood cells had escaped. Sometimes there were small aggregates of polymorphonuclear leucocytes, but glial reaction was absent (Fig. 6). Numerous sections across the left posterior cerebral artery and its branches showed no thrombosis and no structural change in the wall of even the smallest branches. The infarct in the thalamus was also of quite recent date. There was rupture of capillaries with escape of red blood cells and a few collections of polymorphonuclear leucocytes, but glial reaction was very slight. There was no inflammatory reaction in the adjacent ventricle or choroid plexus. The motor fibres of the 
left side were but slightly affected in the region of the thalamic infarct. At a slightly lower level some fibres were involved by ædema and reactive change resulting from the recent thrombosis of the choroid plexus of the obliterated inferior horn of the lateral ventricle. This had involved more the inferior aspect of the corpus striatum and the structures of the posterior part of the internal capsule. There were a few areas of petechial hæmorrhage but no glial reaction as the fibres sweep downwards into the peduncles.

The ependyma of the inferior horn of the left ventricle was preserved in parts, but inflammatory reaction involved the ventricular aspect of the uncus and the hippocampus. The capillary endothelial cells were swollen and red blood cells

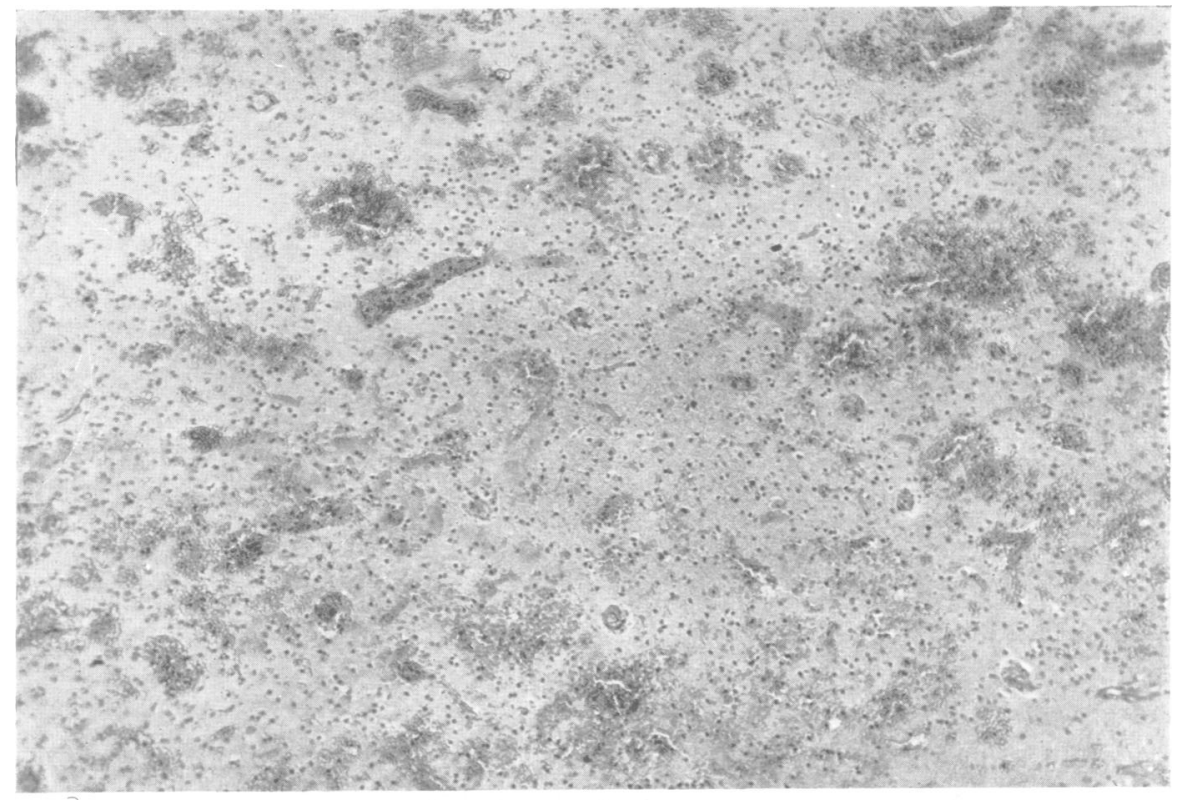

Fig. 6.-The cortex below the calcarine fissure. Glial reaction is absent, but there is some swelling of the endothelial cells of capillaries and an escape of red blood cells.

and sometimes polymorphonuclear leucocytes surrounded some of the capillaries. Microglial cells showed swelling and proliferation. There was no inflammation or vascular lesion in the underlying optic tract and only slight thickening of the pia and a few mononuclear inflammatory cells on the surface of the uncal and hippocampal gyri, and around the cerebral peduncles and midbrain. Nerve cells were slightly reduced in number in the left hippocampal gyrus. On both sides the grooving by the edge of the tentorium has resulted in a localized reduction of nerve cells with necrotic changes in some cell nuclei, and especially on the right side, some proliferation of the microglia.

Apart from slight odema and congestion of vessels, sections through the corpora quadrigemina and the subthalamic nuclei presented no other lesion. The periaqueductal grey matter also appeared normal. Elsewhere in the midbrain the changes were confined to the right side. Where the peduncle was grooved by the tentorium cerebelli there was some œdema, axis cylinders being 
swollen and tortuous, and there was slight proliferation of glial elements, chiefly of the microglia. The changes were not confined to the more superficial areas. There were also areas of necrosis with the bulbous ends of interrupted axis cylinders situated deeper within the peduncle. In areas in the tegmentum and red nucleus the venules and capillaries' were congested and the endothelial lining cells prominent and there were small petechial hæmorrhages without glial reaction.

In the visual pathway the most marked change affected the fibres in front of the chiasma. Degeneration of myelin had occurred in the upper fibres of the right optic nerve and in a wedge-shaped area on the lateral aspect of the left. Behind the chiasma there was no significant loss of fibres. There was no demonstrable change in the third nerve nuclei or in the proximal part of the nerve. There was no degeneration in the fourth cranial nerve, either before or after it came into relation with the edge of the tentorium as it sweeps round the mid-brain.

\section{Commentary}

The main interest lies in the production of lesions in areas remote from the primary lesion by changes in their blood supply. While some of the demonstrable lesions are almost terminal, their distribution indicates those areas which must suffer the greatest functional disturbances by such an alteration of their blood supply. In the occipital lobe the area most affected is that above the calcarine fissure which is supplied by the calcarine and parieto-occipital twigs of the occipital branch of the posterior cerebral artery. In this case the posterior cerebral artery has been affected in its cortical rather than ganglionic distribution. Occlusion was probably only temporary and there was no change in the vessels themselves and no thrombi in their lumen. Moore and Stern (1938) also found no change in even the smallest arterioles. Not all branches were affected equally nor at the same time as the different ages of the infarcts show. Reflex vaso-motor constriction cannot be excluded but the distribution indicates that small arteries, rather than arterioles, must have been affected at the same period.

It is very readily assumed that the posterior cerebral artery in these cases is forced into contact with a free edge of the tentorium (Jefferson 1938, Moore \& Stern 1938). A study of the relationship of the artery and of the tentorial groove (Fig. 1) suggests that while the artery is displaced on to the more exposed inferior surface of the brain by the stretching out of the uncal and hippocampal gyri, it is only in the more posterior part of the course that it comes into relation with the edge of the tentorium. Here, however, its termination, the occipital branch about to enter the calcarine fissure is no more exposed than the course of the anterior and middle temporal branches which are given off earlier. The areas supplied by these vessels were normal but may have been better nourished by anastomotic channels. It is also highly improbable that the ganglionic branches, apparently affected in some of Moore \& Stern's cases, could be thus involved. Some degree of herniation of the uncus is not uncommon and in 
examining uncomplicated cases the possibility that direct pressure by the tentorial edge could affect the vessel appears somewhat unlikely. Especially with rapidly progressive herniation the vessel or its branches may be stretched or distorted and branches kinked. This would result from their situation on the lateral aspect of the bulging uncus in the fissure between the uncal and hippocampal gyri when this carries them away from the mid-line and downwards through the incisura tentorii (Fig. 4). The vessels would readily stretch to accommodate themselves to their new course and might be expected to show no microscopical changes. The brain substance supplied would be irreparably damaged. The herniation affects those parts of the uncal and hippocampal gyri which normally lie just above and medial to the foramen. These are " rolled out" as the space filling lesion above drives them down through the incisura. The wider area of the affected side overlying the foramen results from the shift of the brain to the opposite side until the crus cerebri is notched by the opposite tentorial edge.

Following the classical account of Beevor (1908 and 1909), confirmed by Shellshear (1920), of the cerebral arterial supply, the area of thalamus involved is in great part supplied from the posterior communicating artery. Traction on the posterior communicating artery, and probably also, alterations in the course of this vessel owing to the shift of the hemisphere, as well as the local œdema of the brain surrounding the abscess may be considered as possible causes of this terminal infarction.

Shortly after the sudden apoplectiform seizure the child appeared completely blind and could neither appreciate light nor hand movements and the pupils were dilated and immobile. Complete testing of the visual fields was, therefore, quite impossible. There was some degree of optic atrophy superimposed on the papillœdema, but histological examination of the optic tracts confirmed the clinical opinion that this was insufficient to account for all the symptoms. The absence of lesions in the region of the optic thalamus, or in the right occipital radiation or lobe, suggest that in this child, at least an impression of complete blindness was created by the loss of the left visual cortex or its association tracts.

The involvement of the right crus cerebri, which clinically only produced a transient change in the left plantar reflex, and the terminal lesions in the right red nucleus resulted from the shifting of the axis of the brain bringing the crus against the edge of the tentorium (Kernohan and Woltman 1929). The lesions have resulted from pressure on vessels entering or emerging from the brain. Direct pressure to the right and left hippocampal cortex and to the surface of the right crus has only produced a very minor local effect.

Histologically there was no evidence of any lesion in the neighbourhood of the aqueduct or the third nerve nuclei and the proximal part of both nerves showed no demonstrable abnormality. The pupillary abnormalities probably resulted from pressure of the herniated uncus on the oculomotor nerve.

It is obvious that familiarity with the occurrence of tentorial herniation and the vascular changes taking place in the territory supplied by the posterior cerebral artery would be of assistance in assessing correctly some apparently 
conflicting clinical signs. In the present case after the sudden apoplectiform seizure and the resulting blindness it was recognized that other lesions must be present as well as the abscess in the temporal lobe. Their nature was not recognized until after autopsy.

\section{Summary}

A case of temporal lobe abscess presenting many diagnostic difficulties is described.

Vascular lesions especially in the occipital lobe, resulted from herniation of the uncal and hippocampal gyri through the incisura tentorii. The possible mechanism of the vascular changes is discussed, and as the onset was sudden and no permanent arterial damage demonstrable, temporary occlusion by stretching and kinking of the posterior cerebral artery is considered as well as the possibility of direct pressure from the edge of the tentorium.

Recognition of the possibility of the condition may be of considerable clinical value in a correct localization of apparently conflicting clinical findings.

We wish to thank Professor J. H. Biggart for much valued help and advice.

\section{REFERENCES}

Beevor, C. E. (1908). Brain, 30, 403.

(1909). Philos. Trans., 200, 1.

Jefferson, G. (1938). Arch. Neurol. Psychiat. Chicago, 40, 857.

Kernohan, J. W., and Woltman, H. W. (1929). Arch. Neurol. Psychiat., 21, 274.

Moore, M. T., and Stern, K. (1938). Brain, 61, 70.

Shellshear, J. L. (1920). J. Anat., 55, 27. 